\title{
Las pacientes jóvenes con infección urinaria recurrente son capaces de autodiagnosticar e iniciar su propio tratamiento
}

Patient-initiated treatment of uncomplicated recurrent urinary tract infections in young women. K. Gupta, T. M. Hooton, P.Roberts; Ann Intern Med 2001; 135:9-16.

\section{Objetivo}

Evaluar la habilidad de las pacientes con historia de infección urinaria (ITU) a repetición para autodiagnosticar y tratar un nuevo episodio, las tasas de curación después del inicio del tratamiento y la satisfacción con esta estrategia.

Diseño

Estudio clínico prospectivo no controlado.

Lugar

Clínica de atención primaria universitaria EEUU.

\section{Participantes}

Mujeres de 18 años o más con antecedentes de al menos dos episodios de ITU en los últimos 12 meses. Se excluyeron pacientes con antecedentes de diabetes, hipertensión, enfermedad renal, un embarazo a término en los últimos 12 meses y alergia a las fluoroquinolonas.

\section{Intervención}

Se instruyó a las mujeres para que recolectaran una muestra de orina estéril ante el inicio de los síntomas de ITU. Luego debían iniciar tratamiento antibiótico con ofloxacina o levofloxacina por tres días. A los 10 y 30 días posteriores al inicio del antibiótico (ATB) debían concurrir a la clínica para una entrevista y cultivos de control.

Además, a aquellas pacientes con cultivos negativos se las evaluaba para eventuales diagnósticos alternativos.

Se entregaron cuestionarios de satisfacción para cada episodio.

\section{Resultados}

Participaron 172 mujeres de las cuales 88 reportaron síntomas, cuantificando en total 172 episodios sintomáticos con inicio de tratamiento antibiótico. Además, 64 mujeres reportaron síntomas menores que no motivaron el inicio del tratamiento ATB y todos resolvieron espontáneamente.

En 144 (84\%) de los casos se aisló un uropatógeno en el cultivo y en $19(11 \%)$ sólo se detectó piuria estéril. Del primer grupo 133 casos resolvieron sin complicaciones y 11 fallaron, requiriendo tratamiento adicional. Se registro sólo 1 caso de pielonefritis.

De las pacientes con piuria estéril 3 no fueron evaluadas, una presentó cervicitis por clamidia y el resto $(n=15)$ fueron categorizados como probables ITUs ya que los síntomas revirtieron con el tratamiento.

9 episodios no presentaron piuria ni cultivos positivos y tampoco se llegó a otros diagnósticos, todos presentaron resolución clínica. En resumen, 159 pacientes evaluaron el cuadro adecuadamente (ITU definidas + ITUs probables [95\%]) y de estos episodios 148 $(86 \%)$ resolvieron con el tratamiento iniciado por las pacientes; 12 $(6.9 \%)$ episodios requirieron tratamiento adicional y $27(15.6 \%)$ fueron reevaluados por persistencia de los síntomas o problemas diagnósticos.

La mayoría de las pacientes reportó gran satisfacción con la estrategia.

\begin{tabular}{l|l|l|l}
\hline & $\mathbf{n}(\%)$ & IC $95 \%$ & Comentario \\
\hline ITU confirmada & $144(84 \%)$ & $77-90 \%$ & 172 episodios sintomáticos \\
\hline Diagnósticos correctos & $159(94 \%)$ & $90-99 \%$ & 169 evaluados \\
\hline Falla clínica & $11(7.63 \%)$ & No reportado & \\
\hline auración a los 10 días & $126(96 \%)$ & $93-99 \%$ & 131 evaluados en seguimiento \\
\hline auración sostenida & $81(98 \%)$ & $94-100 \%$ & 83 evaluados al mes \\
\hline
\end{tabular}

$\mathrm{n}$ episodios totales $=172 \mathrm{n}$ episodios evaluados $=169$

\section{Conclusiones}

Las pacientes con antecedentes de ITU a repetición son confiables en diagnosticar y tratar episodios subsecuentes. Esta estrategia es segura y la mayoría de las pacientes reportaron satisfacción con la estrategia.

\section{Comentario}

En este estudio la mayoría de las mujeres con antecedentes de ITU fueron capaces de diagnosticar y tratar sus episodios de ITU posteriores en forma exitosa.

Una de las limitaciones para aplicar este estudio a la práctica clínica es que las pacientes eran todas jóvenes y con un alto nivel de educación. Por otra parte, no existió un grupo control, aunque a los fines del objetivo del estudio el diagnóstico sintomático que realizaban las pacientes era comparado al resultado del urocultivo. No es posible calcular valores de sensibilidad y especificidad para la maniobra de autodetección de ITU ya que 64 pacientes no realizaron el urocultivo porque se encontraban asintomáticas. Aún así, este estudio puede ser discutido desde dos ópticas diferentes.

Para aquellos médicos que han seguido a sus pacientes con ITUs a repetición, y conocen su grado de alarma y responsabilidad, esta es una estrategia segura. En este contexto, este médico está confiando y delegando en su paciente la responsabilidad de tratar su enfermedad. Este tipo de situaciones son muy enriquecedoras para la relación médico-paciente, el médico enseña a su paciente cómo manejar la enfermedad pero a su vez le da autonomía para hacerlo. De esta manera se fortalece la relación entre ellos, se ahorra tiempo y dinero, y queda instalada una idea de colaboración mutua.

Por otra parte, en el contexto de planificar estrategias de salud poblacionales, es importante destacar que las pacientes que más presentan el cuadro de ITU a repetición son las mujeres jóvenes sexualmente activas. Para las pacientes que están alejadas del sistema sanitario, estos episodios son a veces el único contacto que permite iniciar controles de salud y consejos preventivos, principalmente en relación a la prevención del embarazo no deseado y las enfermedades de transmisión sexual. En este sentido, la estrategia de autodiagnóstico y tratamiento de las ITU recurrentes debería ser considerada por los médicos desde su consultorio con sus pacientes en forma individual y no ser planteada como estrategia poblacional. De otra manera posiblemente perderíamos la oportunidad de ofrecer mejores cuidados a una población en riesgo.

Dra. Alejandrina Lo Sasso [ Unidad de Medicina Familiar y Preventiva. Hospital Italiano de Buenos Aires ] 